Received: 06.08 .2018

Revised: 10.06 .2019

Accepted: 28.06 .2019

DOI: $10.17804 / 2410-9908.2019 .3 .006-015$

\title{
CONVERGENCE PATTERNS OF KRYLOV SUBSPACE SOLVERS IN THE SIMULATION OF LARGE ELASTIC-PLASTIC DEFORMATIONS OF HETEROPHASE MEDIA
}

\author{
Yu. V. Khalevitsky ${ }^{\text {a)* }}$, A. V. Konovalov ${ }^{\text {b) }}$ and A. S. Partin ${ }^{\text {c) }}$ \\ Institute of Engineering Science, Ural Branch of the Russian Academy of Sciences, \\ 34 Komsomolskaya St., 620049, Ekaterinburg, Russian Federation \\ a) (iD https://orcid.org/0000-0001-8176-9871 me@dijkstra.ru; \\ b) iD https://orcid.org/0000-0001-9131-8636 avk@imach.uran.ru; \\ c) iD https://orcid.org/0000-0002-9279-9213 $ه$ lmd@imach.uran.ru \\ *Corresponding author. E-mail: me@dijkstra.ru \\ Address for correspondence: 34 Komsomolskaya St., 620049, Ekaterinburg, Russian Federation \\ Tel.: +7 (343) 3623026
}

Simulating the process of heterophase medium deformation is essential for the scientific substantiation of composite material forming. Representative-volume models of composite materials are generally represented as a conglomerate of elastic and elastic-plastic bodies. Usually, composite deformations are numerically simulated by the finite element method, which requires solving a series of simultaneous linear equations. When applying a fine mesh, an iterative Krylov subspace solver is usually used.

We present a comparison of convergence for several iterative solvers in a two-dimensional finite-element model problem on the deformation of a heterophase representative volume of a composite. The volume contains 13,000 three-node triangular finite elements. The composite is based on the AMg6 alloy, and it contains 10 vol\% of silicon carbide reinforcement.

Six methods are compared within the scope of the article: the biconjugate gradient stabilized method (BiCGStab), the generalized minimal residual method (GMRES), the conjugate gradient squared method (CGS), the quasi minimal residual method (QMR) and variants of the BiCGStab and QMR methods, namely BiCGStab(L) and TFQMR. The best timing is shown by a relatively rare QMR method.

Keywords: linear solver, finite element method, Krylov subspace solver, elastic-plastic deformation, composite material, representative volume.

\section{Acknowledgments}

The work was supported from the federal budget (government registration No. AAAA-A18118020790140-5) in terms of studying linear solver convergence in elastoplastic problems and partially supported by the Russian Science Foundation (Project 14-19-01358) in the part of developing a finite element suite for simulating composite structure deformation.

\section{References}

1. Mishnaevsky L. Computational mesomechanics of composites: Numerical analysis of the effect of microstructures of composites of strength and damage resistance. London, John Wiley and Sons, 2007, 294 p. ISBN: 978-0-470-02764-6. 
2. Schmauder S., Mishnaevsky L. Micromechanics and nanosimulation of metals and composites: Advanced methods and theoretical concepts. Heidelberg, Springer, 2008, 420 p., ISBN 978-3540-78678-8.

3. Pugacheva N.B., Michurov N.S., Bykova T.M. The structure and properties of the 30AL-70SIC metal matrix composite material. Diagnostics, Resource and Mechanics of materials and structures, 2015, iss. 6, pp. 6-18. DOI: 10.17804/2410-9908.2015.6.006-018.

4. Smirnov S.V., Konovalov A.V., Myasnikova M.V., Khalevitskiy Yu.V., Smirnov A.S., Igumnov A.S. A Computational Model of V95/SiCp (7075/ SiCp) Aluminum Matrix Composite Applied to Stress-Strain State Simulation under Tensile, Compressive and Shear Loading Conditions. Diagnostics, Resource and Mechanics of materials and structures, 2017, iss. 6, pp. 16-27. DOI: 10.17804/2410-9908.2017.6.016-027.

5. Pozdeev A.A., Trusov P.V., Nyashin Yu.I. Bolshie uprugoplasticheskie deformatsii: teoriya, algoritmy, prilozheniya [Large elasticplastic deformation: theory, algorithms and applications]. Moscow, Nauka, 1986, 232 p. (In Russian).

6. Yousef S. Iterative Methods for Sparse Linear Systems. Second Edition. SIAM Publishing, 2003, 547 p. DOI: 10.1137/1.9780898718003.

7. Driscoll Tobin A., Chuan Toh Kim, Trefethen Lloyd Nicholas. Matrix iterations: the six gaps between potential theory and convergence. Theory Center technical report. Cornell Theory Center, Cornell University, 1996. (https://books.google.ru/books?id=HbFQAAAAYAAJ).

8. Khalevitsky Yu.V., Konovalov A.V., and Partin A.S. On convergence of various iterative linear solvers in heterophase elastoplastic media deformation models. In: AIP Conference Proceedings, 2018, vol. 2053, pp. 030026. DOI: 10.1063/1.5084387.

9. Noël M.N., Satish C.R., Lloyd N.T. How Fast are Nonsymmetric Matrix Iterations? SIAM Journal on Matrix Analysis and Applications, 1992, vol. 13, no. 3, pp. 778-795. DOI: 10.1137/0613049.

10. Ilyin V.P. Finite Element Methods and Technologies. In: Parallelnye vychislitelnye tekhnologii (PaVT'2009): trudy mezhdunarodnoy konferentsii [Parallel Computational Technologies: International Conference Proceedings]. Novosibirsk, ICM\&MG Publ., 2007. (In Russian).

11. Smirnov A.S., Shveikin V.P., Smirnova E.O., Belozerov G.A., Konovalov Anatoly V., Vichuzhanin Dmitry I., Muizemnek Olga Yu. Effect of silicon carbide particles on the mechanical and plastic properties of the AlMg6/10\% SiC metal matrix composite. Journal of Composite Materials, 2018, vol. 52 (24), pp. 3351-3363. DOI: 10.1177/0021998318765622.

12. Askeland D., Fulay P., Wright W. The Science and Engineering of Materials. Cengage Learning, 2010. ISBN 978-1-4899-2895-5.

13. Van der Vorst H.A. Bi-CGSTAB: A Fast and Smoothly Converging Variant of Bi-CG for the Solution of Nonsymmetric Linear Systems. SIAM J. Sci. and Stat. Comput., 1992, vol. 13, iss. 2, pp. 631-644. DOI: 10.1137/0913035.

14. Youcef S., Martin H.S. GMRES: A Generalized Minimal Residual Algorithm for Solving Nonsymmetric Linear Systems. SIAM J. Sci. and Stat. Comput., 1986, vol. 7, iss. 3, pp. 856-869. DOI: $10.1137 / 0907058$.

15. Freund R.W., Nachtigal N.M. QMR: a quasi-minimal residual method for non-Hermitian linear systems. Numerische Mathematik, 1991, vol. 60, iss. 1, pp. 315-339. DOI: 10.1007/BF01385726.

16. Sonneveld Peter. CGS, A Fast Lanczos-Type Solver for Nonsymmetric Linear systems. SIAM Journal on Scientific Computing, 1989, vol. 10, no. 1, pp. 36-52.

17. Sleijpen G.L.G., Fokkema D.R. BiCGstab(L) for linear equations involving matrices with complex spectrum. Electron. Trans. Numer. Anal., 1993, no 1, pp. 11-32.

18. Freund R.W. A transpose-free quasi-minimal residual algorithm for non-Hermitian linear systems. SIAM J. Sci. Stat. Comput., 1993, vol. 14, pp. 470-482. 
19. Zhang J. Preconditioned Krylov subspace methods for solving nonsymmetric matrices from CFD applications. Computer Methods in Applied Mechanics and Engineering, 2000, vol. 189, iss. 3, pp. 825-840.

20. Khalevitsky Yu.V., Burmasheva N.V., Konovalov A V., and Partin A.S. Comparative study of Krylov Subspace method implementations for a GPU cluster in elastoplastic problems. In: AIP Conference Proceedings, 2016, vol. 1785, pp. 040024. DOI: 10.1063/1.4967081. 
Подана в журнал: 06.08.2019

УДК 519.683:539.3

DOI: $10.17804 / 2410-9908.2019 .3 .006-015$

\title{
ПАТТЕРНЫ СХОДИМОСТИ КРЫЛОВСКИХ МЕТОДОВ В МОДЕЛИРОВАНИИ БОЛЬШИХ УПРУГОПЛАСТИЧЕСКИХ ДЕФОРМАЦИЙ ГЕТЕРОФАЗНОЙ СРЕДЫ
}

\author{
Ю. В. Халевицкий ${ }^{\text {a* }}$, А. В. Коновалов ${ }^{\text {()) }}$, А. С. Партин ${ }^{\text {B) }}$ \\ Федеральное государственное бюджетное учреждение науки \\ Институт машиноведения Уральского отделения Российской академии наук, \\ ул. Комсомольская, 34, Екатеринбург, Российская Федераиия \\ a) (iD https://orcid.org/0000-0001-8176-9871 me@dijkstra.ru; \\ б) iD https://orcid.org/0000-0001-9131-8636 avk@imach.uran.ru; \\ в) (iD https://orcid.org/0000-0002-9279-9213 1md@imach.uran.ru \\ *Ответственный автор. Электронная почта: me@ dijkstra.ru \\ Адрес для переписки: ул. Комсомольская, 34, 620049 г. Екатеринбург, Российская Федерация \\ Тел.: +7 (343) 362-30-26
}

Моделирование процесса деформации гетерофазных сред необходимо для научного обоснования технологических процессов обработки давлением композиционных материалов. Модели представительных объемов этих материалов представляют собой конгломерат упругих и упругопластических тел. Численное моделирование деформирования заготовок из композиционных материалов осуществляется методом конечных элементов, при использовании которого необходимо многократно решать ряд систем линейных алгебраических уравнений (СЛАУ). При решении задач с мелкой сеткой, как правило, используются итерационные методы решения СЛАУ, основанные на подпространствах Крылова.

В работе для сетки, содержащей 13 тыс. треугольных трехузловых конечных элементов, получена СЛАУ в двумерной модельной конечно-элементной задаче деформирования гетерофазного представительного объема металломатричного композита на основе сплава АМг6 и 10 об. \% частиц карбида кремния.

Проведены численные эксперименты решения данной СЛАУ шестью итерационными методами: стабилизированным методом бисопряженных градиентов (BiCGStab), обобщенным методом минимальных невязок (GMRES), методом квазиминимальных невязок (QMR), методом квадратичных сопряженных градиентов (CGS), вариантом метода BiCGStab BiCGStab(L) и вариантом метода QMR - TFQMR. Сравнение времени решения СЛАУ этими методами показывает, что наилучшие результаты достигаются при использовании метода QMR.

Ключевые слова: система линейных алгебраических уравнений, метод конечных элементов, метод подпространств Крылова, упругопластическая деформация, композит, представительный объем.

\section{1. Введение}

Разработка технологических процессов обработки давлением металломатричных композиционных материалов (ММК) является новой и актуальной задачей для инженеров. Назначение научно обоснованных режимов технологического процесса должно опираться на результаты компьютерного моделирования эволюции напряженно-деформированного состояния металломатричного композита в процессе его деформирования как на макроуровне, так и на уровне представительного микрообъема $[1,2]$. В силу наличия в металлической матрице 
композита упрочняющих частиц напряженно-деформированное состояние в микрообъеме внутри компонентов его внутренней структуры существенно неоднородно [3], хотя на макроуровне оно может быть однородным [4].

В статье рассмотрена модель металломатричного композита АМг6/10 \% $\mathrm{SiC}$ с матрицей из сплава АМг6 и 10 об. \% содержанием упрочняющих частиц карбида кремния $\mathrm{SiC}$, изготовленного по порошковой технологии. Микроструктура композита содержит гранулы пластичного материала матрицы, окруженные конгломератом из частиц карбида кремния и оплавленного металла (рис. 1 a). Такой конгломерат называется далее прослойкой.

Эффективным инструментом компьютерного моделирования эволюции напряженнодеформированного состояния представительного объема ММК при развитых пластических деформациях является метод конечных элементов, основанный на принципе виртуальной мощности в скоростной форме [5].

Метод подразумевает многократное решение ряда систем линейных алгебраических уравнений (СЛАУ), причем каждая следующая система зависит от предыдущей. Для рассматриваемой модели материала матрицы коэффициентов СЛАУ несимметричные (имеют симметричный портрет), разреженные, ленточные (значительно разрежены внутри ленты), блочные (с размером блока, соответствующим размерности задачи) имеют значительную размерность и плохо обусловлены. Несимметричность матриц возникает в результате использования в определяющих соотношениях для упругопластической среды ковариантной производной тензора напряжений Коши. Портрет и структура матрицы типичны для метода конечных элементов. Размерность матрицы определяется количеством узлов пространственной дискретизации. Моделирование деформации представительного объема рассмотренного композита (с тонкой прослойкой) требует использования миллионов конечных элементов, при этом матрица имеет размерность порядка миллионов. Для такой размерности матрицы и принятых свойств материала ММК число ее обусловленности имеет порядок $10^{6}$. Количество СЛАУ, которые необходимо решить в рамках одной задачи, определяется временной дискретизацией. Шаг по времени достаточно мал и выбирается таким образом, чтобы обеспечить устойчивость решения упругопластической задачи при больших искажениях формы сетки в процессе изменения нагрузки во времени. Поэтому в рассматриваемой задаче необходимо решить в общей сложности порядка $10^{4}$ систем. Каждая следующая СЛАУ формируется с использованием предыдущего решения, поэтому параллельное решение нескольких систем не представляется возможным, однако системы, возникающие в рамках одного шага по нагрузке, имеют одинаковую матрицу жесткости. Это позволяет повторно использовать предобуславливатели или даже полную факторизацию матрицы системы.

В силу большой разреженности внутри ленты, программы, основанные на прямых методах решения СЛАУ, быстро исчерпывают оперативную память компьютера. Даже если система обладает достаточным объемом памяти, решение прямым методом происходит значительно медленнее, чем итерационным, несмотря на возможность повторного использования факторизации. Поэтому для решения систем уравнений с такими матрицами, как правило, применяются современные итерационные методы решения СЛАУ, основанные на подпространствах Крылова [6]. Сходимость таких методов определяется спектральными свойствами матрицы $[7,8]$, которые в свою очередь зависят от используемой модели материала. В силу сложности построения аналитических оценок для комбинации множества численных методов, использующихся при решении задачи, выбор наиболее подходящего метода решения СЛАУ для каждой новой модели материала необходимо осуществлять экспериментально [9].

Ранее в работе [10] на основе вычислительных экспериментов была проанализирована производительность итерационных методов CGS, TFQMR, BiCGStab и GMRES. B нашей статье дополнительно к ним рассмотрены методы QMR и BiCGStab(L). Полученные результаты обосновывают выбор метода решения СЛАУ в методе конечных эле- 
ментов для моделирования процессов развитой пластической деформации представительных объемов металломатричных композитов.

\section{2. Модельная задача и методы решения}

Вычислительные эксперименты проводили на матрице СЛАУ, полученной из модельной осесимметричной задачи пластического деформирования двухмерного представительного объема ММК АМг6/10 \% SiC с матрицей из сплава АМг6 и 10 об. \% содержанием упрочняющих частиц карбида кремния $\mathrm{SiC}$, изготовленного по порошковой технологии. Микроструктура композита, содержащая гранулы пластичного материала матрицы, окруженные конгломератом (прослойкой) из частиц карбида кремния и оплавленного металла, представлена на рис. 1 a. Изображение конечно-элементной сетки представительного объема рассматриваемого композита приведено на рис. 1 б. Конечно-элементная сетка получена трассировкой границ гранул в программе GetData Graph Digitizer. Результаты трассировки были обработаны специальной программой и импортированы в расчетную программу. Гранулы металла матрицы обозначены голубым цветом, а прослойка - сиреневым.

Прослойка состоит в основном из упрочняющих частиц, однако за счет их соединения с оплавленным металлом матрицы прослойка сохраняет пластичность, поэтому в модели представительного объема материал прослойки считаем однородной сплошной средой. Материал гранул и прослойки рассматриваем как изотропную и изотропно упрочняющуюся упругопластическую среду со своими упругими и пластическими свойствами, подверженную большим пластическим деформациям. Среда подчиняется ассоциированному закону пластического течения с функцией текучести Мизеса.

Зависимости сопротивления деформации от величины эквивалентной деформации материала матрицы, а также композита в целом, взяты из работы [11]. Свойства прослойки были определены по правилу фаз [12] из экспериментальных данных [11]. Полученные кривые сопротивления деформации в зависимости от эквивалентной деформации представлены на рис. 2. Упругие константы были взяты из справочной литературы и вычислены по правилу фаз: для АМг6-модуль Юнга 93 ГПа, коэффициент Пуассона 0,3; для прослойки - модуль Юнга 120 ГПа, коэффициент Пуассона 0,34.

Вычислительная конечно элементная модель деформирования представительного объема ММК построена на принципе виртуальной мощности в приращениях перемещений. Скоростная составляющая вариационного равенства данного принципа построена согласно работе [5]. В силу геометрической и физической нелинейности процесса деформирования представительного объема ММК нагрузка прикладывалась малыми шагами для обеспечения устойчивости решения упругопластической задачи. Сетка содержала 13 тыс. треугольных трехузловых конечных элементов. Моделировали сжатие плоскими плитами без трения представительного объема со степенью обжатия $\Delta h / h_{0}=0,5$ где $h_{0}$ и $\Delta h$ - высота представительного объема и ее изменение соответственно. В конце обжатия в материале матрицы композита имела место развитая пластическая деформация с величиной эквивалентной деформации, достигающей значения 1,6. Полученная при этом матрица коэффициентов СЛАУ, а также вектор ее правой части были выгружены во вторичную память компьютера в формате, пригодном для работы с математическим пакетом MATLAB, установленном на суперкомпьютере «Уран» ИММ УрО РАН. Дальнейшие вычислительные эксперименты провели с использованием пакета MATLAB. 


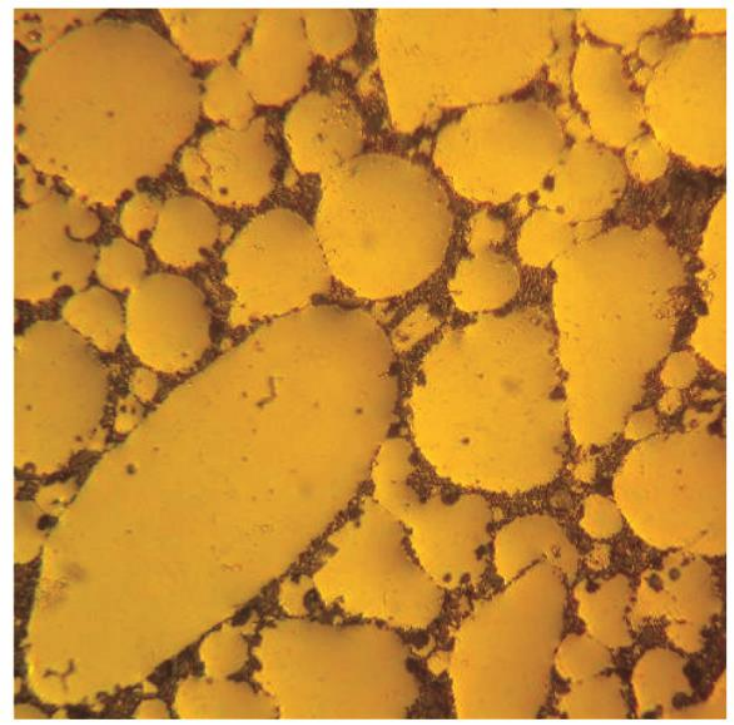

$a$

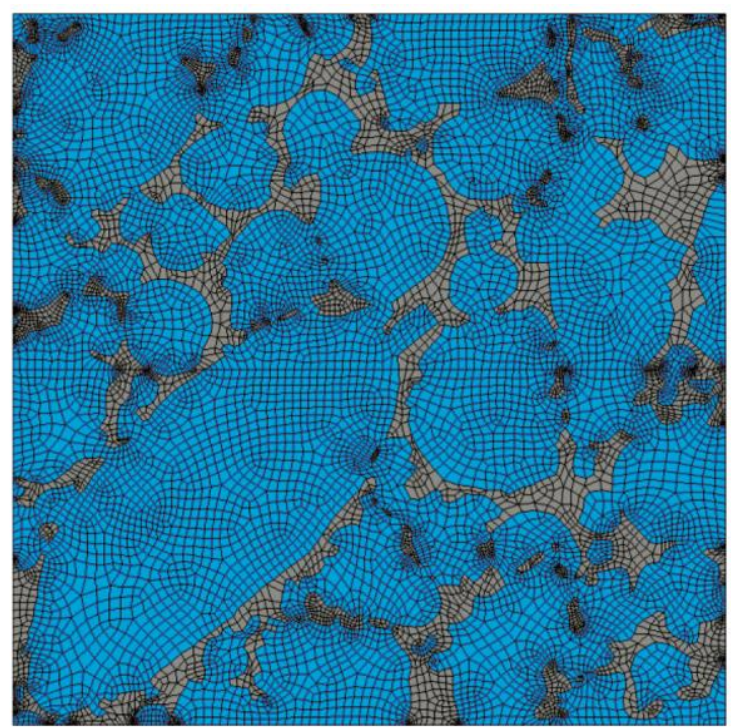

$\sigma$

Рис. 1. Изображение микроструктуры ММК АМг6/10 \% SiC (a) и построенная по изображению микроструктуры конечно-элементная сетка (б)

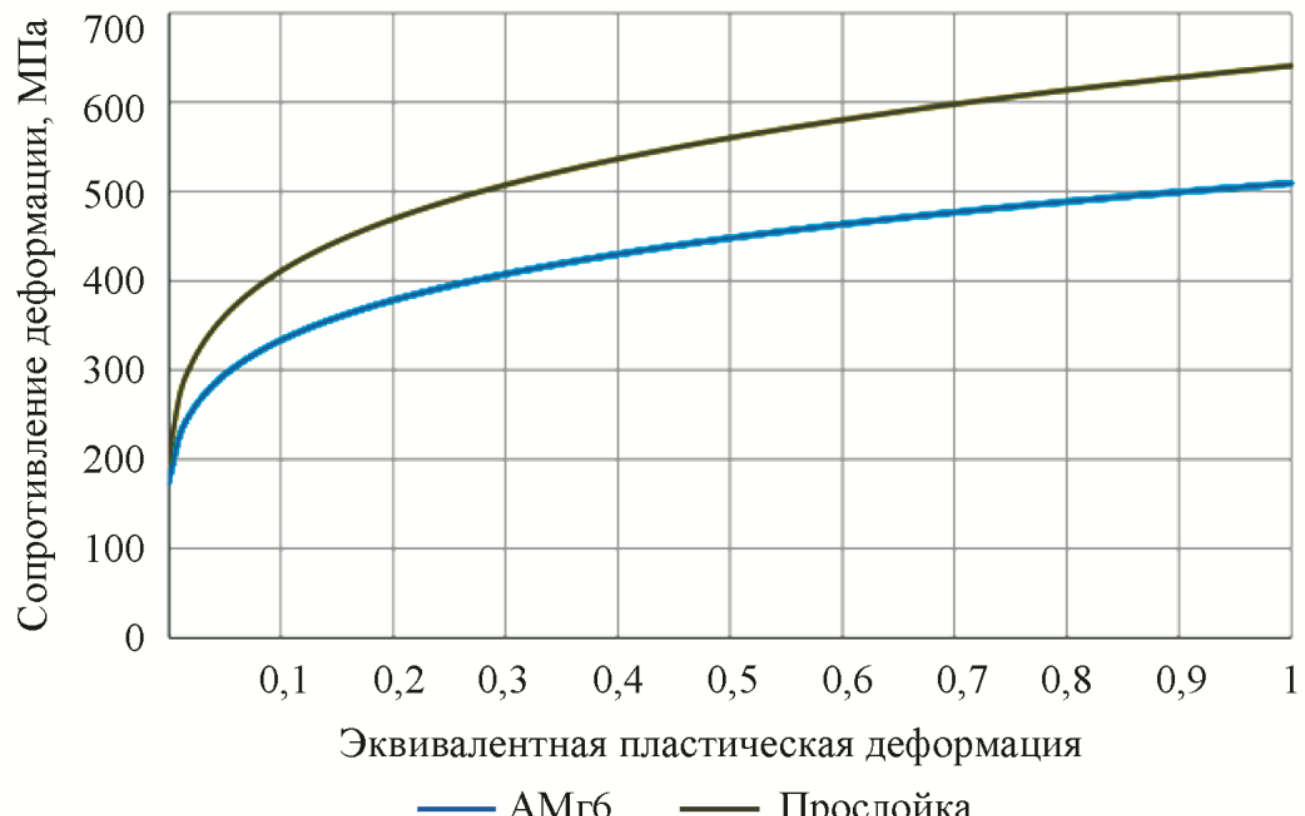

Рис. 2. Сопротивление деформации составляющих композита

Исследовали распространенные методы решения СЛАУ: стабилизированный метод бисопряженных градиентов (BiCGStab) [13], обобщенный метод минимальных невязок (GMRES) [14], метод квазиминимальных невязок (QMR) [15], метод квадратичных сопряженных градиентов (CGS) [16], а также варианты методов BiCGStab и QMR: BiCGStab(L) [17] и TFQMR [18]. В процессе вычислений измеряли астрономическое время достижения критерия сходимости $r / r_{0}=10^{-6}$, где $r$ и $r_{0}-$ невязка на текущей и первой итерациях решения СЛАУ соответственно. Также фиксировали невязку на каждой из итераций. 


\section{3. Результаты и обсуждение}

К сожалению, имеющими в распоряжении авторов вычислительными ресурсами не удалось определить число обусловленности использованной матрицы СЛАУ из-за ее большой размерности. Оценка числа обусловленности с помощью подпрограммы condest пакета MATLAB дает величину порядка $10^{6}$, однако, вероятно, число обусловленности гораздо больше.

Bce решения рассмотренными методами, за исключением метода TFQMR, сошлись. Ниже представлено время решения СЛАУ разными методами.

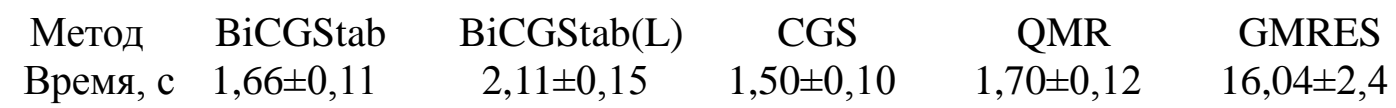

Видно, что наименьшее время решения СЛАУ обеспечивают методы QMR и CGS. Meтод GMRES затрачивает на решение наибольшее время. Это касается и метода BiCGStab(L), который также использует итерации, основанные на минимальной невязке [17]. В рассматриваемой задаче использование таких итераций не приводит к улучшению сходимости.
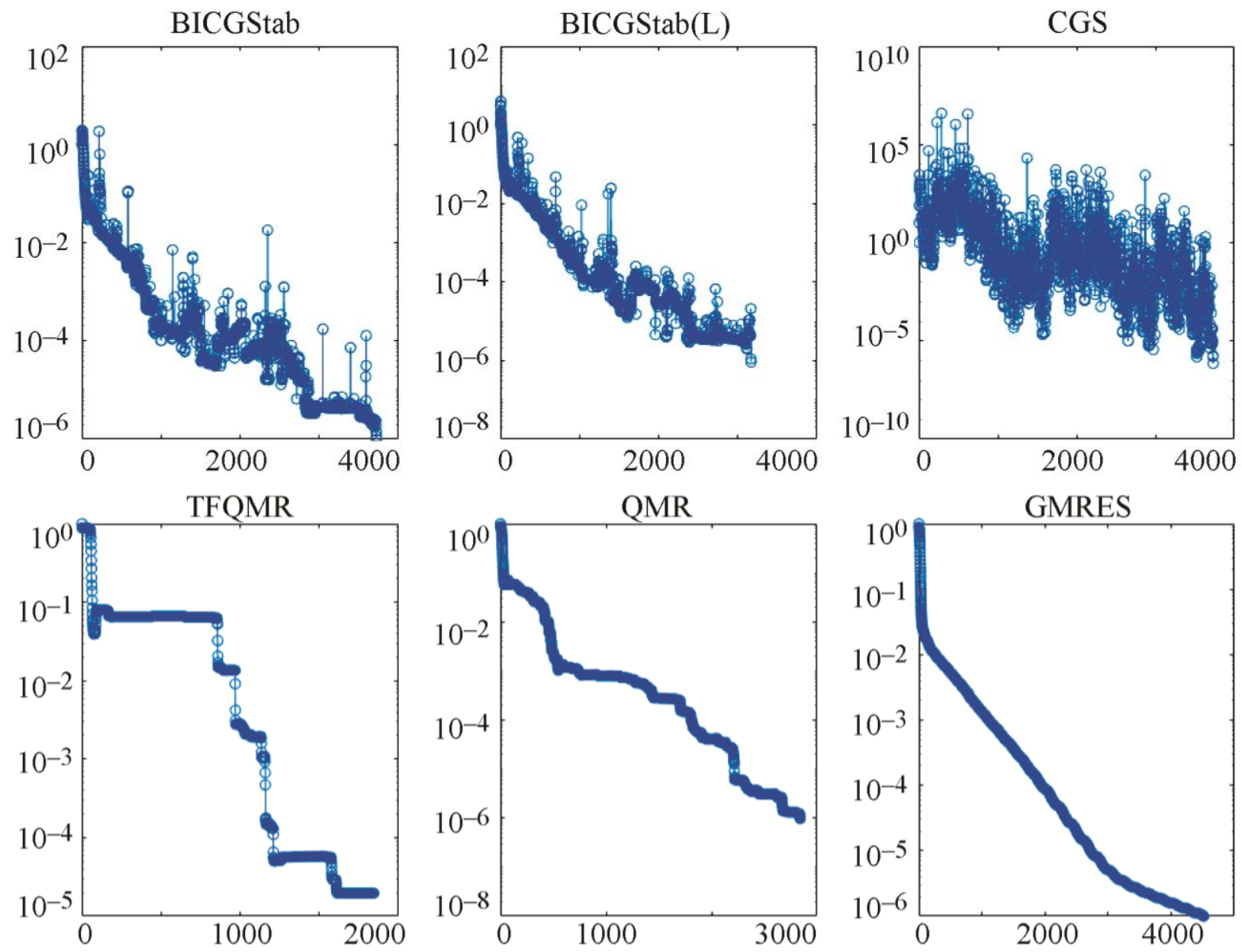

Рис. 3. Зависимость нормы невязки методов решения СЛАУ от номера итерации (снимок экрана программного комплекса MATLAB)

Зависимость нормы невязки каждого из рассмотренных методов от номера итерации представлена на рис. 3. При использовании методов QMR и GMRES невязка монотонно убывает с каждой следующей итерацией. При использовании методов BiCGStab и CGS норма 
невязки не убывает монотонно и подвержена значительным осцилляциям, которые могут составлять несколько порядков, при этом сходимость достигается на одной из осцилляций. Метод TFQMR подвержен стагнациям [19], и его график изменения невязки имеет характерный «лестничный» вид (рис. 3). Метод не достигает сходимости в рассматриваемой задаче из-за стагнации. При этом метод TFQMR достигает сходимости с простыми предобуславливателями при моделировании деформации однородной среды с матрицами значительно большей размерности [20].

Метод QMR значительно менее распространен в сравнении с методами GMRES и BiCGStab, однако показывает в данной задаче наилучший результат.

\section{4. Заключение}

Метод GMRES не подходит для использования в рассматриваемом классе задач. Методы CGS и QMR обеспечивают практически одинаковое время решения СЛАУ, однако метод CGS подвержен сильным осцилляциям нормы невязки. В рассматриваемой задаче метод TFQMR не сходится. Методы BiCGStab и BiCGStab(L) показывают сходное с методом QMR время решения СЛАУ, однако также подвержены осцилляциям и не могут быть рекомендованы для рассматриваемого класса задач. По результатам вычислительных экспериментов можно рекомендовать для использования в конечно элементных расчетах напряженнодеформированного состояния представительных объемов металломатричных композитов относительно малораспространенный метод QMR.

\section{Благодарность}

Работа выполнена по бюджетной теме № АAAA-A18-118020790140-5 в части исследования сходимости итерационных методов решения СЛАУ в упругопластической задаче и при частичной финансовой поддержке гранта РНФ (Проект № 14-19-01358) в части разработки конечно-элементного программного комплекса для моделирования процессов деформации композитных материалов.

\section{Литература}

1. Mishnaevsky L. Computational mesomechanics of composites: Numerical analysis of the effect of microstructures of composites of strength and damage resistance. - London : John Wiley and Sons, 2007. - 294 p. - ISBN: 978-0-470-02764-6.

2. Schmauder S., Mishnaevsky L. Micromechanics and nanosimulation of metals and composites: Advanced methods and theoretical concepts. - Heidelberg : Springer, 2008. - 420 p. ISBN 978-3-540-78678-8.

3. Pugacheva N. B., Michurov N. S., Bykova T. M. The structure and properties of the 30AL-70SIC metal matrix composite material // Diagnostics, Resource and Mechanics of materials and structures. - 2015. - Iss. 6. - P. 6-18. - DOI: 10.17804/2410-9908.2015.6.006-018.

4. A Computational Model of V95/SiCp (7075/ SiCp) Aluminum Matrix Composite Applied to Stress-Strain State Simulation under Tensile, Compressive and Shear Loading Conditions / S. V. Smirnov, A. V. Konovalov, M. V. Myasnikova, Yu. V. Khalevitskiy, A. S. Smirnov, A. S. Igumnov // Diagnostics, Resource and Mechanics of materials and structures. - 2017. - Iss. 6. P. 16-27. - DOI: 10.17804/2410-9908.2017.6.016-027.

5. Поздеев А. А., Трусов П. В., Няшин Ю. И. Большие упругопластические деформации: теория, алгоритмы, приложения. - М. : Наука, 1986. - 232 с.

6. Yousef S. Iterative Methods for Sparse Linear Systems. Second Edition. - SIAM Publishing, 2003. - 547 p. - DOI: 10.1137/1.9780898718003. 
7. Driscoll T. A., Toh K. C., Trefethen L .N. Matrix iterations: the six gaps between potential theory and covergence // Theory Center technical report. Cornell Theory Center, Cornell University. - 1996. URL: https://books.google.ru/books?id=HbFQAAAAYAAJ

8. Khalevitsky Yu. V., Konovalov A. V., Partin A. S. On convergence of various iterative linear solvers in heterophase elastoplastic media deformation models // AIP Conference Proceedings. 2018. - Vol. 2053. - P. 030026. - DOI: 10.1063/1.5084387.

9. Noël M. N., Satish C. R., Lloyd N. T. How Fast are Nonsymmetric Matrix Iterations? // SIAM Journal on Matrix Analysis and Applications. - 1992. - Vol. 13, no. 3. - P. 778-795. DOI: $10.1137 / 0613049$.

10. Ильин В. П. Проблемы высокопроизводительных технологий решения больших разреженных СЛАУ // Параллельные вычислительные технологии (ПаВТ’2009) : труды международной научной конференции. - Челябинск : Издательский центр ЮУрГУ, 2009.

11. Effect of silicon carbide particles on the mechanical and plastic properties of the AlMg6/10 $\%$ SiC metal matrix composite / A. S. Smirnov, V. P. Shveikin, E. O. Smirnova, G. A. Belozerov, Anatoly V. Konovalov, Dmitry I. Vichuzhanin, Olga Yu. Muizemnek // Journal of Composite Materials. - 2018. - Vol. 52 (24). - P. 3351-3363. - DOI: 10.1177/0021998318765622.

12. Askeland D., Fulay P., Wright W. The Science and Engineering of Materials. - Cengage Learning, 2010. - ISBN 978-1-4899-2895-5.

13. Van der Vorst H. A. Bi-CGSTAB: A Fast and Smoothly Converging Variant of Bi-CG for the Solution of Nonsymmetric Linear Systems // SIAM J. Sci. and Stat. Comput. - 1992. Vol. 13, iss. 2. - P. 631-644. - DOI: 10.1137/0913035.

14. Youcef S., Martin H. S. GMRES: A Generalized Minimal Residual Algorithm for Solving Nonsymmetric Linear Systems // SIAM J. Sci. and Stat. Comput. - 1986. - Vol. 7, iss. 3. P. 856-869. - DOI: 10.1137/0907058.

15. Freund R. W., Nachtigal N. M. QMR: a quasi-minimal residual method for non-Hermitian linear systems // Numerische Mathematik. - 1991. - Vol. 60, iss. 1. - P. 315-339. DOI: $10.1007 / \mathrm{BF} 01385726$.

16. Sonneveld P. CGS, A Fast Lanczos-Type Solver for Nonsymmetric Linear systems // SIAM Journal on Scientific Computing. - 1989. - Vol. 10, no. 1. - P. 36-52.

17. Sleijpen G. L. G., Fokkema D. R. BiCGstab(L) for linear equations involving matrices with complex spectrum // Electron. Trans. Numer. Anal. - 1993. - No. 1. - P. 11-32.

18. Freund R. W. A transpose-free quasi-minimal residual algorithm for non-Hermitian linear systems // SIAM J. Sci. Stat. Comput. - 1993. - Vol. 14. - P. 470-482.

19. Zhang J. Preconditioned Krylov subspace methods for solving nonsymmetric matrices from CFD applications // Computer Methods in Applied Mechanics and Engineering. - 2000. Vol. 189, iss. 3. - P. 825-840.

20. Comparative study of Krylov Subspace method implementations for a GPU cluster in elastoplastic problems / Yu. V. Khalevitsky, N. V. Burmasheva, A. V. Konovalov, A. S. Partin // AIP Conference Proceedings. - 2016. - Vol. 1785. - P. 040024. - DOI: 10.1063/1.4967081. 